ZOHAIB, A. ${ }^{1 *}$

ANJUM, S.A. ${ }^{1}$

JABBAR, A. ${ }^{1}$

TABASSUM, T. ${ }^{1}$

ABBAS, T. ${ }^{1}$

NAZIR, U.

* Corresponding author:

<alizohaib208@gmail.com>

Received: February 27, 2016

Approved: May 11, 2016

Planta Daninha 2017; v35:e017160380

\section{Allelopathic Effect of leguminous Weeds on Rate, SyNCHRONIZATION AND TIME OF GERMinAtion, AND Biomass Partitioning in Rice}

\section{Efeito Alelopático de Plantas Daninhas Leguminosas na Taxa, Sincronização e Tempo de Germinação e Partição de Biomassa em Plantas de Arroz}

ABSTRACT - The present study was aimed at determining the allelopathic influence of water extracts at varying concentrations $(2.5 \%$ and $5 \% \mathrm{w} / \mathrm{v})$ and residues with varying decomposition periods $(0,15$ and 30 days) at $2 \%(\mathrm{w} / \mathrm{w})$ concentration of four weeds (Trigonella polycerata, Vicia sativa, Lathyrus aphaca, Medicago polymorpha) against rate, synchronization and time of germination, and biomass partitioning of rice through plant and soil bioassays. Germination and biomass partitioning in rice plants were inhibited by extracts and residues. The maximum decrease in energy of germination (88.6\%) and increase in time for $50 \%$ germination (210.4\%) were caused by the L. aphaca extract at $5 \%$ concentration as compared to control. Root and shoot, fresh weight (13.6 and 28.6\%, respectively) and dry weight (20.4 and $41.7 \%$, respectively) were reduced by the M. polymorpha extract at $5 \%$ concentration. The Vicia sativa extract at both concentrations caused a great increase in the root/shoot ratio (125-145.8\%). Trigonella polycerata and $V$. sativa residues decomposed for 30 days caused maximum inhibition of energy of emergence (71.4\%) and their decomposition for zero and 15 days, respectively, inhibited coefficient of uniformity of emergence (78.9\%). Medicago polymorpha and L. aphaca residues decomposed for 30 days exaggerated time for $50 \%$ emergence (47.1 and 50.3\%, respectively) when compared with control. Medicago polymorpha residues decomposed for 30 days exerted the greatest inhibitory effect on shoot fresh weight (46.1\%) and root dry weight (59.9\%). Vicia sativa with 30 days and T. polycerata residues without decomposition produced the least root fresh weight $(65.3 \%)$ and shoot dry weight (52.6\%), respectively. The root/shoot ratio (56.8\%) was increased by T. polycerata residues without decomposition. Phenolics from leguminous weeds caused inhibition of germination/emergence and biomass partitioning in rice. Therefore, these weeds must be eradicated from the field at their initial growth stages.

Keywords: allelopathy, leguminous weeds, rice, germination, biomass partitioning.

RESUMO - O presente estudo teve como objetivo determinar a influência alelopática de extratos aquosos em concentrações variadas $(2,5 \%$ e $5 \% \mathrm{p} / \mathrm{v})$ e resíduos com periodos de decomposição diferentes (0, 15 e 30 dias) na concentração de $2 \%(p / v)$ de quatro plantas daninhas (Trigonella polycerata, Vicia sativa, Lathyrus aphaca e Medicago polymorpha) na taxa, sincronização e tempo de germinação e partição de biomassa de arroz através de bioensaios vegetais e em solo. A germinação e a partição de biomassa nas plantas de arroz foram inibidas pelos extratos e resíduos. A redução máxima de energia de germinação $(88,6 \%)$ e o aumento no tempo de $50 \%$ de germinação $(210,4 \%)$ foram causados pelo extrato de $\boldsymbol{L}$. aphaca na concentração de $5 \%$, em relação ao controle. Houve

1 Department of Agronomy, University of Agriculture, Faisalabad, Pakistan 38040. 
redução, na raiz e parte aérea, do peso fresco $(13,6 \%$ e $28,6 \%$, respectivamente) e do peso seco $(20,4 \%$ e 41,7\%, respectivamente) pelo extrato de M. polymorpha na concentração de $5 \%$. O extrato de V. sativa em ambas as concentrações causou grande aumento na relação raiz/parte aérea (125-145,8\%). Os resíduos de Trigonella polycerata e $\boldsymbol{V}$. sativa decompostos por 30 dias causaram a inibição máxima de energia de emergência $(71,4 \%)$, e a respectiva decomposição por zero e 15 dias aumentou o coeficiente de inibição de uniformidade de emergência (78,9\%). Os resíduos de Medicago polymorpha e L. aphaca decompostos por 30 dias aumentaram o tempo para 50\% de emergência (47,1\% e 50,3\%, respectivamente), quando comparados com o controle. Os residuos de Medicago polymorpha decompostos por 30 dias exerceram o maior efeito inibitório sobre o peso fresco da parte aérea $(46,1 \%)$ e o peso seco da raiz $(59,9 \%)$. Os resíduos de Vicia sativa com 30 dias e os de T. polycerata sem decomposição resultaram no menor peso fresco da raiz $(65,3 \%)$ e peso seco da parte aérea $(52,6 \%)$, respectivamente. O aumento da relação raiz/parte aérea $(56,8 \%)$ foi provocado por resíduos de T. polycerata sem decomposição. Os compostos fenólicos de plantas daninhas leguminosas causaram a inibição da germinação/emergência e partição de biomassa nas plantas de arroz. Portanto, as plantas daninhas devem ser erradicadas do campo nos estádios iniciais de desenvolvimento.

Palavras-chave: alelopatia, plantas daninhas leguminosas, arroz, germinação, partição de biomassa.

\section{INTRODUCTION}

The direct or indirect stimulatory or inhibitory effects of plants on each other through the release of chemicals into the environment are referred to as allelopathy (Rice, 1984). The substances released are named as allelochemicals and are mostly secondary metabolites in nature (Asaduzzaman et al., 2010). Allelochemicals belong to various classes depending upon their chemical properties. The most commonly occurring allelochemicals in plants are terpenes, fatty acids, phenolics and indoles (Noguchi, 2008). Germination events as well as growth processes of plants are checked upon by allelochemicals via perturbation of various physiological processes such as photosynthesis, respiration, cell division and enlargement, enzyme activity, protein synthesis and metabolic activities (Lin et al., 2004). It has been known that different plant species impose differential allelopathic effect on recipient plant species (Hamayun et al., 2005). Allelochemicals are released from plants by various modes such as exudation, leaching, volatilization and decomposition (Narwal et al., 2005). In field conditions, the mode of release of allelochemicals is generally residue decomposition (Singh et al., 2001). In addition, allelochemicals have a differential behavior as regards their concentration when imposing allelopathic effects on recipient plants. Higher concentrations are associated with imposition of inhibitory effects while lower concentrations exhibit stimulatory allelopathic effects on germination and seedling growth (Hossain and Alam, 2010).

Yellow vetchling (Lathyrus aphaca), bur clover (Medicago polymorpha), common vetch (Vicia sativa) and trefoil (Trigonella polycerata) are weeds commonly found in temperate and tropical regions of the world, e.g., in Europe, Africa, the United States, Australia and many Asian countries including India and Pakistan. These weeds are associated with various winter season crops including barley, wheat, lentil, gram, and winter season vegetables; however, they are commonly found in wheat (Hussain et al., 1985; Ahmad and Shaikh, 2003). These are leguminous in nature, belong to family Fabaceae and are winter annual weeds. They complete their life cycle from October to March and leave their residues in the field, thus releasing allelochemicals in the soil, during decomposition. Substantial losses are caused by these weeds in the rice-wheat cropping system. Research reports have described the allelopathic effect of $V$. sativa (Mukhtar and Bajwa, 2011; Zohaib et al., 2014a), L. aphaca (Om et al., 2002), T. polycerata (Zohaib et al., 2014b) and $M$. polymorpha (Khan et al., 2012) on the germination and growth processes of plants. These weeds contain various phenolic compounds viz. L. aphaca (gallic acid, m-coumaric acid, caffeic acid, syringic acid), M. polymorpha (m-coumaric acid, vanilic acid, p-coumaric acid, 4-hydroxy-3-methoxybenzoic acid), T. polycerata (4-hydroxy-3-methoxybenzoic acid, m-coumaric acid, syringic acid) and $V$. sativa (p-coumaric acid, 4-hydroxy-3-methoxybenzoic acid, ferulic acid) (Zohaib et al., 2014a,b). Previous studies have shown their allelopathic effect on germinability, seedling growth and vigor of rice (Zohaib et al., 2014b). The present study was accomplished to 
evaluate the allelopathic influence of water extracts of $L$. aphaca, $V$. sativa, T. polycerata and $M$. polymorpha at varying concentrations and their decomposing residues with different decomposition durations on synchronization, rate and time of germination/emergence, and biomass partitioning in rice plants.

\section{MATERIALS AND METHODS}

A set of experiments was carried out to assess the allelopathic effect of $L$. aphaca, M. polymorpha, V. sativa and T. polycerata on germination and biomass partitioning in rice in a laboratory at Department of Agronomy, University of Agriculture, Faisalabad, Pakistan. Residues of $L$. aphaca, V. sativa, M. polymorpha and T. polycerata were collected by uprooting the plants from fields at maturity during winter 2013. Plants residues were dried at room temperature, i.e., $25{ }^{\circ} \mathrm{C}$ to get the maximum recovery of allelochemicals present in weed plants. Small pieces of all the weed plants ranging in size from 1 to $3 \mathrm{~cm}$ were made using a pair of scissors. The pieces were further dried in an oven at $70^{\circ} \mathrm{C}$ for 24 hours. The pieces of whole plants were soaked in distilled water for 24 hours in a ratio of 1:20 (w/v) at room temperature. The water extracts of all leguminous weeds were filtered through a sterile metal tea strainer with a mesh size of $0.5 \mathrm{~mm}$ followed by filtration by Whattman No. 1 filter paper to obtain the pure aqueous extracts without impurities. The extracts prepared in this manner were used as stock solutions with concentration of $5 \%(\mathrm{w} / \mathrm{v})$, and further dilutions to $2.5 \%(\mathrm{w} / \mathrm{v})$ of extracts of all weeds were made by adding distilled water to the stock solution for carrying out the experiment at varying concentrations. The aqueous extracts were stored in separate bottles and tagged for bioassay studies.

Two experiments were conducted using water extracts and residues of weed plants. The experiments were conducted using a completely randomized design and a two-factor arrangement in each experiment and four replications.

\section{Experiment 1: Allelopathic effect of water extracts of weeds on germination and biomass partitioning in rice seedlings}

The assessment of the allelopathic effect of water extracts of weeds viz. V. sativa, L. aphaca, $M$. polymorpha, and T. polycerata was carried out at varying concentrations viz. $2.5 \%(1: 40 \mathrm{w} / \mathrm{v})$ and $5 \%(1: 20 \mathrm{w} / \mathrm{v})$ against rice. Ten rice seeds were placed on a filter paper in $9 \mathrm{~cm}$ diameter petri dishes, separately. In each Petri dish, $7 \mathrm{~mL}$ of aqueous extracts and distilled water was poured according to the nature of the treatment. Distilled water was used as control for comparison. The temperature during the growing period of rice ranged from 29.0 to $31.8{ }^{\circ} \mathrm{C}$. The experiment was observed for 16 days. Petri dishes were kept moistened during the whole period of study.

\section{Experiment 2: Allelopathic effect of weed residues on emergence and biomass partitioning in rice seedlings}

Residues of $L$. aphaca, M. polymorpha, $V$. sativa and $T$. polycerata at $2 \%(\mathrm{w} / \mathrm{v})$ concentration were incorporated in the soil and allowed to decompose for varying duration viz. 0,15 and 30 days. Soil with no weed residues was used as control. Ten seeds of rice were sown in each pot. Distilled water was used to keep the soil moist during the whole period of the experiment. The temperature during the whole experiment ranged from $32.5-37.0^{\circ} \mathrm{C}$. The experiment was observed for 16 days. The number of seeds germinated/emerged was counted on a daily basis following the procedure of seedling evaluation in the Handbook of the Association of Official Seed Analysts (AOSA, 1990). When root size reached $2 \mathrm{~mm}$, the seed was counted as germinated. Energy of germination/ emergence was determined according to Farooq et al. (2006);

Energy of germination /emergence $=\left(\frac{\text { No. seeds germinated } / \text { emerged } 4 \text { days after sowing }}{\text { Total No. of seeds tested }}\right) \times 100$

Coefficient of uniformity of germination/emergence was calculated using the formula of Bewley and Black (1994); 


$$
\text { Coefficient of uniformity of germination/emergence }=\frac{\sum n i}{\left(M G T-t_{i}\right)^{2} \times n i}
$$

where, $n_{i}=$ Number of seeds completing emergence on day $t_{i}$, MGT $=$ Mean emergence time, $t_{i}=$ Time in days, starting on the day of sowing to the day of first emergence

The formula of Ellis and Roberts (1981) was used to calculate the mean germination/ emergence time:

$$
\text { MGT }=\frac{\sum \mathrm{Dn}}{\mathrm{N}}
$$

where, $n$ is the number of seeds germinated or seedlings emerged on day $D$ and $N$ is the total number of germinated/emerged seeds.

Time to reach $50 \%$ germination/emergence was calculated according to the following formula of Coolbear et al. (1984) modified by Farooq et al. (2005):

$$
T_{50}=t_{i}+\left[\frac{N / 2-n_{i}}{n_{j}-n_{i}}\right]\left(t_{j}-t_{i}\right)
$$

where, $N$ is the number of germinated/emerged seeds, and $n_{i}$ and $n_{j}$ are the cumulative number of seeds germinated/emerged by adjacent counts at times $t_{i}$ and $t_{j}$, respectively, when $n_{i}<N / 2<n_{j}$.

Shoots and roots of rice seedlings were weighed to determine fresh weight, followed by oven drying at $70{ }^{\circ} \mathrm{C}$ to determine dry weight. The weights were expressed in milligrams. Root/shoot ratio of rice seedlings was determined by using the following formula;

$$
\text { Root } / \text { shoot ratio }=\frac{\text { Root dry weight }}{\text { Shoot dry weight }}
$$

The data collected was analyzed using Fisher's analysis of variance and treatments' means were compared by using the least significant difference test at $\mathrm{p} \geq 0.05$ (Steel et al., 1997).

\section{RESULTS AND DISCUSSION}

\section{Germination bioassay}

The results revealed that water extracts of leguminous weeds imposed significant inhibitory allelopathic effect on germination and biomass partitioning in rice seedlings (Table 1 and 2). Water extracts of all weeds at different concentrations imposed an inhibitory effect on energy of germination and time to reach 50\% germination; however, coefficient of uniformity of germination was not affected. Vicia sativa and L. aphaca extracts at $5 \%$ concentration showed maximum inhibition of energy of germination $(88.6 \%)$ of rice seeds while there were statistically similar results for water extracts of $T$. polycerata at $5 \%$ concentration and $M$. polymorpha at $2.5 \%$ as well as $5 \%$ concentration when compared with control. Maximum time to 50\% germination (207.4\%) was perceived by the impact of the water extract of $L$. aphaca at $5 \%$ concentration as compared to control. Furthermore, the effect of the $V$. sativa extract at $5 \%$ concentration on time to reach $50 \%$ germination was statistically similar with that of $L$. aphaca at $5 \%$ concentration (Table 1 ). Root and shoot, fresh weight (13.6 and 28.6\%, respectively) and dry weight (20.4 and 41.7\% respectively) were reduced mostly by the influence of the $M$. polymorpha extract at $5 \%$ concentration when compared with control. However, shoot and root fresh weights as well as root dry weight were increased by the L. aphaca extract at 5\% concentration when compared with control. Furthermore, root/shoot ratio of rice seedlings was increased by the weed water extracts as compared to control. The highest root/shoot ratio (125-145.8\%) of rice plants occurred by the influence of the $V$. sativa water extract at both concentrations (Table 1 and 2). 
ZOHAIB, A. et al. Allelopathic effect of leguminous weeds on rate, synchronization and time of germination, and biomass ...

Table 1 - Allelopathic effect of the water extract of leguminous weeds on germination and shoot fresh weight of rice

\begin{tabular}{|c|c|c|c|c|c|c|c|c|}
\hline \multirow[t]{2}{*}{ Treatment } & \multicolumn{2}{|c|}{$\begin{array}{c}\text { Energy of germination } \\
(\%)\end{array}$} & \multicolumn{2}{|c|}{$\begin{array}{l}\text { Coefficient of uniformity } \\
\text { of germination }\end{array}$} & \multicolumn{2}{|c|}{$\begin{array}{c}\text { Time to reach } 50 \% \\
\text { germination } \\
\text { (days) }\end{array}$} & \multicolumn{2}{|c|}{$\begin{array}{l}\text { Shoot fresh weight } \\
\text { (mg) }\end{array}$} \\
\hline & $2.5 \%$ & $5 \%$ & $2.5 \%$ & $5 \%$ & $2.5 \%$ & $5 \%$ & $2.5 \%$ & $5 \%$ \\
\hline Control & $87.50 \mathrm{a}$ & $87.50 \mathrm{a}$ & 2.61 & 2.61 & $1.89 \mathrm{f}$ & $1.89 \mathrm{f}$ & $7.21 \mathrm{~b}$ & $7.21 \mathrm{~b}$ \\
\hline$V$. sativa & $\begin{array}{c}52.50 \mathrm{~b} \\
(-40)\end{array}$ & $\begin{array}{l}10.00 \mathrm{c} \\
(-88.6)\end{array}$ & $\begin{array}{c}0.75 \\
(-71.3)\end{array}$ & $\begin{array}{c}0.15 \\
(-94.3)\end{array}$ & $\begin{array}{l}2.64 \mathrm{def} \\
(39.7)\end{array}$ & $\begin{array}{c}6.00 \mathrm{a} \\
(217.5)\end{array}$ & $\begin{array}{c}6.41 \mathrm{~cd} \\
(-11.1)\end{array}$ & $\begin{array}{l}6.77 b c \\
(-6.1)\end{array}$ \\
\hline T. polycerata & $\begin{array}{l}50.00 \mathrm{~b} \\
(-42.9)\end{array}$ & $\begin{array}{l}12.50 \mathrm{c} \\
(-85.7)\end{array}$ & $\begin{array}{c}0.74 \\
(--71.6)\end{array}$ & $\begin{array}{c}1.31 \\
(-49.8)\end{array}$ & $\begin{array}{l}2.79 \mathrm{de} \\
(47.6)\end{array}$ & $\begin{array}{l}3.94 \mathrm{bc} \\
(108.5)\end{array}$ & $\begin{array}{l}6.88 \text { bc } \\
(-4.6)\end{array}$ & $\begin{array}{l}6.46 \mathrm{~cd} \\
(-10.4)\end{array}$ \\
\hline L. aphaca & $\begin{array}{l}57.50 b \\
(-34.3)\end{array}$ & $\begin{array}{l}10.00 \mathrm{c} \\
(-88.6)\end{array}$ & $\begin{array}{c}0.57 \\
(-78.2)\end{array}$ & $\begin{array}{c}0.96 \\
(-63.2)\end{array}$ & $\begin{array}{l}2.52 \text { ef } \\
(33.3)\end{array}$ & $\begin{array}{c}5.81 \mathrm{a} \\
(207.4)\end{array}$ & $\begin{array}{c}6.05 \mathrm{~d} \\
(-16.1)\end{array}$ & $\begin{array}{l}8.00 \mathrm{a} \\
(11.0)\end{array}$ \\
\hline M. polymorpha & $\begin{array}{l}20.00 \mathrm{c} \\
(-77.1)\end{array}$ & $\begin{array}{l}15.00 \mathrm{c} \\
(-82.9)\end{array}$ & $\begin{array}{c}0.43 \\
(-83.5)\end{array}$ & $\begin{array}{c}0.49 \\
(-81.2)\end{array}$ & $\begin{array}{l}3.38 \mathrm{~cd} \\
(78.8)\end{array}$ & $\begin{array}{l}4.63 \mathrm{~b} \\
(145)\end{array}$ & $\begin{array}{c}5.91 \mathrm{~d} \\
(-18.0)\end{array}$ & $\begin{array}{c}5.15 \mathrm{e} \\
(-28.6)\end{array}$ \\
\hline LSD at $p \leq 0.05$ & \multicolumn{2}{|c|}{12.01} & \multicolumn{2}{|c|}{ NS } & \multicolumn{2}{|c|}{0.780} & \multicolumn{2}{|c|}{0.689} \\
\hline
\end{tabular}

Means with different letters from each other differ significantly at $\mathrm{p} \leq 0.05$; NS $=$ Non-significant; negative values in parenthesis represent a percent reduction while positive values represent a percent increase over control.

Table 2 - Allelopathic effect of the water extract of leguminous weeds on biomass partitioning of rice

\begin{tabular}{|c|c|c|c|c|c|c|c|c|}
\hline \multirow[t]{2}{*}{ Treatment } & \multicolumn{2}{|c|}{$\begin{array}{l}\text { Root fresh weight } \\
(\mathrm{mg})\end{array}$} & \multicolumn{2}{|c|}{$\begin{array}{c}\text { Shoot dry weight } \\
(\mathrm{mg})\end{array}$} & \multicolumn{2}{|c|}{$\begin{array}{l}\text { Root dry weight } \\
\text { (mg) }\end{array}$} & \multicolumn{2}{|c|}{ Root/shoot ratio } \\
\hline & $2.5 \%$ & $5 \%$ & $2.5 \%$ & $5 \%$ & $2.5 \%$ & $5 \%$ & $2.5 \%$ & $5 \%$ \\
\hline Control & $2.35 \mathrm{~d}$ & $2.35 \mathrm{~d}$ & $5.83 \mathrm{a}$ & $5.83 \mathrm{a}$ & $1.37 \mathrm{~d}$ & $1.37 \mathrm{~d}$ & $0.24 \mathrm{~d}$ & $0.24 \mathrm{~d}$ \\
\hline V. sativa & $\begin{array}{l}3.49 \mathrm{~b} \\
(48.5)\end{array}$ & $\begin{array}{c}4.09 \mathrm{a} \\
(74.0) \\
\end{array}$ & $\begin{array}{c}3.99 \mathrm{c} \\
(-31.6) \\
\end{array}$ & $\begin{array}{c}4.21 \mathrm{c} \\
(-27.8) \\
\end{array}$ & $\begin{array}{l}2.14 \mathrm{~b} \\
(56.2)\end{array}$ & $\begin{array}{l}2.48 \mathrm{a} \\
(81.0) \\
\end{array}$ & $\begin{array}{r}0.54 \mathrm{a} \\
(125.0) \\
\end{array}$ & $\begin{array}{r}0.59 \mathrm{a} \\
(145.8) \\
\end{array}$ \\
\hline T. polycerata & $\begin{array}{l}3.01 \mathrm{c} \\
(28.1)\end{array}$ & $\begin{array}{l}2.20 \mathrm{de} \\
(-6.4)\end{array}$ & $\begin{array}{r}5.00 \mathrm{~b} \\
(-14.2) \\
\end{array}$ & $\begin{array}{l}4.66 b \\
(-20.1) \\
\end{array}$ & $\begin{array}{l}1.47 \mathrm{~d} \\
(7.3)\end{array}$ & $\begin{array}{c}1.06 \mathrm{e} \\
(-22.6) \\
\end{array}$ & $\begin{array}{l}0.29 \mathrm{~cd} \\
(20.8)\end{array}$ & $\begin{array}{l}0.23 \mathrm{~d} \\
(-4.2) \\
\end{array}$ \\
\hline L. aphaca & $\begin{array}{c}3.29 b c \\
(40)\end{array}$ & $\begin{array}{l}4.31 \mathrm{a} \\
(83.4)\end{array}$ & $\begin{array}{c}4.20 \mathrm{c} \\
(-28.0)\end{array}$ & $\begin{array}{l}5.58 \mathrm{a} \\
(-4.3)\end{array}$ & $\begin{array}{l}1.78 \mathrm{c} \\
(29.9)\end{array}$ & $\begin{array}{l}2.35 \mathrm{a} \\
(71.5)\end{array}$ & $\begin{array}{c}0.43 \mathrm{~b} \\
(79.2)\end{array}$ & $\begin{array}{l}0.42 \mathrm{~b} \\
(75.0)\end{array}$ \\
\hline M. polymorpha & $\begin{array}{l}3.30 \mathrm{bc} \\
(40.4)\end{array}$ & $\begin{array}{c}2.03 \mathrm{e} \\
(-13.6)\end{array}$ & $\begin{array}{c}3.95 \mathrm{c} \\
(-32.2)\end{array}$ & $\begin{array}{c}3.40 \mathrm{~d} \\
(-41.7)\end{array}$ & $\begin{array}{l}1.83 \mathrm{c} \\
(33.6)\end{array}$ & $\begin{array}{c}1.09 \mathrm{e} \\
(-20.4)\end{array}$ & $\begin{array}{l}0.46 \mathrm{~b} \\
(91.7)\end{array}$ & $\begin{array}{l}0.32 \mathrm{c} \\
(33.3)\end{array}$ \\
\hline LSD at $p \leq 0.05$ & \multicolumn{2}{|c|}{0.313} & \multicolumn{2}{|c|}{0.404} & \multicolumn{2}{|c|}{0.204} & \multicolumn{2}{|c|}{0.069} \\
\hline
\end{tabular}

Means with different letters from each other differ significantly at $\mathrm{p} \leq 0.05$; NS $=$ Non-significant; negative values in parenthesis represent a percent reduction while positive values represent a percent increase over control.

\section{Pot study}

Residues of all leguminous weeds inhibited emergence and biomass partitioning in rice seedlings with all decomposition durations (Table 3 and 4). Energy of emergence and coefficient of uniformity of emergence of rice were decreased while time to $50 \%$ emergence was increased by the impact of weed residues. There was maximum inhibition of energy of emergence (71.4\%) by residues of $L$. aphaca without decomposition and residues of $T$. polycerata, $V$. sativa and $M$. polymorpha with a period of 30 days of decomposition. The minimum coefficient of uniformity of emergence $(78.9 \%)$ of rice was perceived by the effect of $T$. polycerata residues without decomposition when compared with control. However, V. sativa residues with 15 days decomposition exerted a similar effect on the coefficient of uniformity of emergence. The maximum increase in time to $50 \%$ emergence $(50.3 \%)$ occurred by the influence of decomposing L. aphaca residues for 30 days, when compared with control. Nonetheless, the effect of $M$. polymorpha and T. polycerata residues with 30 days decomposition was similar to that of L. aphaca residues with 30 days decomposition on time to reach $50 \%$ emergence (Table 3 ). The lowest shoot fresh weight $(46.1 \%)$ and root fresh weight $(65.3 \%)$ of rice seedlings were observed 
by the influence of the $M$. polymorpha and $V$. sativa residues with 30 days decomposition, respectively. However, the lowest shoot dry weight (52.6\%) and root dry weight (59.9\%) of rice seedlings were caused by the influence of the T. polycerata residues without decomposition and the residues of $M$. polymorpha with 30 days decomposition, when compared with control, respectively. A differential behavior was observed for root/shoot ratio of rice seedlings. There was a differential response of the root/shoot ratio by the influence of the weed residues. The root/shoot ratio was increased by the residues of some weeds while it was decreased by others as compared to control. The maximum root/shoot ratio $(56.8 \%)$ was perceived by the effect of T. polycerata residues without decomposition and the lowest root/shoot ratio $(54.5 \%)$ was found by the influence of the residues of $L$. aphaca with 30 days decomposition (Tables 3 and 4).

Table 3 - Allelopathic effect of residues of leguminous weeds on emergence and shoot fresh weight of rice

\begin{tabular}{|c|c|c|c|c|c|c|c|c|c|c|c|c|}
\hline \multirow[t]{2}{*}{ Treatment } & \multicolumn{3}{|c|}{$\begin{array}{c}\text { Energy of emergence } \\
(\%)\end{array}$} & \multicolumn{3}{|c|}{$\begin{array}{c}\text { Coefficient of uniformity of } \\
\text { emergence }\end{array}$} & \multicolumn{3}{|c|}{$\begin{array}{c}\text { Time to reach } 50 \% \text { emergence } \\
\text { (days) }\end{array}$} & \multicolumn{3}{|c|}{$\begin{array}{l}\text { Shoot fresh weight } \\
(\mathrm{mg})\end{array}$} \\
\hline & 0 days & 15 days & 30 days & 0 days & 15 days & 30 days & 0 days & 15 days & 30 days & 0 days & 15 days & 30 days \\
\hline Control & $35.00 \mathrm{a}$ & $35.00 \mathrm{a}$ & $35.00 \mathrm{a}$ & $0.19 \mathrm{~b}$ & $0.19 \mathrm{~b}$ & $0.19 \mathrm{~b}$ & $5.99 \mathrm{e}$ & $5.99 \mathrm{e}$ & $5.99 \mathrm{e}$ & $28.05 \mathrm{~b}$ & $28.05 \mathrm{~b}$ & $28.05 \mathrm{~b}$ \\
\hline V. sativa & $\begin{array}{l}22.50 b c \\
(-35.7)\end{array}$ & $\begin{array}{l}15.00 \text { cde } \\
(-57.1)\end{array}$ & $\begin{array}{l}10.00 \mathrm{e} \\
(-71.4)\end{array}$ & $\begin{array}{l}0.06 \mathrm{de} \\
(-68.4)\end{array}$ & $\begin{array}{c}0.04 \mathrm{e} \\
(-78.9)\end{array}$ & $\begin{array}{l}0.17 b \\
(-10.5)\end{array}$ & $\begin{array}{l}6.69 \text { cde } \\
(11.7)\end{array}$ & $\begin{array}{l}8.44 \mathrm{ab} \\
(40.9)\end{array}$ & $\begin{array}{l}7.63 \mathrm{bc} \\
(27.4)\end{array}$ & $\begin{array}{l}24.20 \mathrm{de} \\
(-13.7)\end{array}$ & $\begin{array}{l}22.79 \text { ef } \\
(-18.8)\end{array}$ & $\begin{array}{l}20.99 \mathrm{fg} \\
(-25.2)\end{array}$ \\
\hline T. polycerata & $\begin{array}{l}20.00 \text { bcd } \\
(-42.9)\end{array}$ & $\begin{array}{l}25.00 \mathrm{~b} \\
(-28.6)\end{array}$ & $\begin{array}{l}10.00 \mathrm{e} \\
(-71.4)\end{array}$ & $\begin{array}{c}0.04 \mathrm{e} \\
(-78.9)\end{array}$ & $\begin{array}{l}0.11 \mathrm{c} \\
(-42.1)\end{array}$ & $\begin{array}{l}0.08 \text { cde } \\
(-57.9)\end{array}$ & $\begin{array}{l}7.25 \mathrm{~cd} \\
(21.0)\end{array}$ & $\begin{array}{l}6.29 \mathrm{de} \\
(5.0)\end{array}$ & $\begin{array}{l}8.88 \mathrm{a} \\
(48.2)\end{array}$ & $\begin{array}{l}20.79 \mathrm{fg} \\
(-25.9)\end{array}$ & $\begin{array}{l}25.22 \mathrm{~cd} \\
(-10.1)\end{array}$ & $\begin{array}{l}19.92 \mathrm{gh} \\
(-29.0)\end{array}$ \\
\hline L. aphaca & $\begin{array}{l}10.00 \mathrm{e} \\
(-71.4)\end{array}$ & $\begin{array}{l}25.00 \mathrm{~b} \\
(-28.6)\end{array}$ & $\begin{array}{l}12.50 \mathrm{de} \\
(-64.3)\end{array}$ & $\begin{array}{l}0.18 b \\
(-5.3)\end{array}$ & $\begin{array}{l}0.09 \mathrm{~cd} \\
(-52.6)\end{array}$ & $\begin{array}{l}0.08 \text { cde } \\
(-57.9)\end{array}$ & $\begin{array}{l}6.94 \text { cde } \\
(15.9)\end{array}$ & $\begin{array}{l}6.56 \mathrm{cde} \\
(9.5)\end{array}$ & $\begin{array}{l}9.00 \mathrm{a} \\
(50.3)\end{array}$ & $\begin{array}{c}30.44 \mathrm{a} \\
(8.5)\end{array}$ & $\begin{array}{l}18.29 \mathrm{hi} \\
(-34.8)\end{array}$ & $\begin{array}{l}17.54 \mathrm{i} \\
(-37.5)\end{array}$ \\
\hline M. polymorpha & $\begin{array}{l}25.00 \mathrm{~b} \\
(-28.6)\end{array}$ & $\begin{array}{l}40.00 \mathrm{a} \\
(14.3)\end{array}$ & $\begin{array}{l}10.00 \mathrm{e} \\
(-71.4)\end{array}$ & $\begin{array}{c}0.16 \mathrm{~b} \\
(-15.8)\end{array}$ & $\begin{array}{l}0.27 \mathrm{a} \\
(42.1)\end{array}$ & $\begin{array}{l}0.07 \text { cde } \\
(-63.2)\end{array}$ & $\begin{array}{l}6.23 \mathrm{de} \\
(4.0)\end{array}$ & $\begin{array}{c}4.23 \mathrm{f} \\
(-29.4)\end{array}$ & $\begin{array}{l}8.81 \mathrm{a} \\
(47.1)\end{array}$ & $\begin{array}{c}26.62 \mathrm{bc} \\
(-5.1)\end{array}$ & $\begin{array}{l}\text { 19.13 ghi } \\
(31.8)\end{array}$ & $\begin{array}{l}15.13 \mathrm{j} \\
(-46.1)\end{array}$ \\
\hline LSD at $p \leq 0.05$ & \multicolumn{3}{|c|}{7.506} & \multicolumn{3}{|c|}{0.046} & \multicolumn{3}{|c|}{1.115} & \multicolumn{3}{|c|}{2.365} \\
\hline
\end{tabular}

Means with different letters from each other differ significantly at $\mathrm{p} \leq 0.05$; NS $=$ Non-significant; negative values in parenthesis represent a percent reduction while positive values represent a percent increase over control.

Table 4 - Allelopathic effect of residues of leguminous weeds on biomass partitioning of rice

\begin{tabular}{|c|c|c|c|c|c|c|c|c|c|c|c|c|}
\hline \multirow[t]{2}{*}{ Treatment } & \multicolumn{3}{|c|}{$\begin{array}{l}\text { Root fresh weight } \\
\text { (mg) }\end{array}$} & \multicolumn{3}{|c|}{$\begin{array}{l}\text { Shoot dry weight } \\
(\mathrm{mg})\end{array}$} & \multicolumn{3}{|c|}{$\begin{array}{l}\text { Root dry weight } \\
\text { (mg) }\end{array}$} & \multicolumn{3}{|c|}{ Root/shoot ratio } \\
\hline & 0 days & 15 days & 30 days & 0 days & 15 days & 30 days & 0 days & 15 days & 30 days & 0 days & 15 days & 30 days \\
\hline Control & $12.12 \mathrm{a}$ & $12.12 \mathrm{a}$ & $12.12 \mathrm{a}$ & $7.34 \mathrm{~b}$ & $7.34 \mathrm{~b}$ & $7.34 \mathrm{~b}$ & $3.19 \mathrm{a}$ & $3.19 \mathrm{a}$ & $3.19 \mathrm{a}$ & $0.44 \mathrm{~cd}$ & $0.44 \mathrm{~cd}$ & $0.44 \mathrm{~cd}$ \\
\hline V. sativa & $\begin{array}{c}6.18 \mathrm{e} \\
(-49.0)\end{array}$ & $\begin{array}{l}4.85 \text { fgh } \\
(-60.0)\end{array}$ & $\begin{array}{r}4.21 \mathrm{~h} \\
(-65.3) \\
\end{array}$ & $\begin{array}{l}.16 \mathrm{fg} \\
(-43.3)\end{array}$ & $\begin{array}{c}5.75 \mathrm{e} \\
(-21.7) \\
\end{array}$ & $\begin{array}{l}6.60 \mathrm{~cd} \\
(-10.1)\end{array}$ & $\begin{array}{l}2.29 \mathrm{de} \\
(-28.2)\end{array}$ & $\begin{array}{l}1.80 \mathrm{gh} \\
(-43.6)\end{array}$ & $\begin{array}{l}3.16 \mathrm{a} \\
(-0.9)\end{array}$ & $\begin{array}{l}0.55 \mathrm{~b} \\
(25.0)\end{array}$ & $\begin{array}{c}0.31 \mathrm{fg} \\
(-29.5)\end{array}$ & $\begin{array}{l}0.48 \mathrm{c} \\
(9.1)\end{array}$ \\
\hline T. polycerata & $\begin{array}{c}9.12 \mathrm{c} \\
(-24.8) \\
\end{array}$ & $\begin{array}{l}10.63 \mathrm{~b} \\
(-12.3)\end{array}$ & $\begin{array}{c}5.63 \text { ef } \\
(-53.5)\end{array}$ & $\begin{array}{c}3.48 \mathrm{~h} \\
(-52.6) \\
\end{array}$ & $\begin{array}{c}8.74 \mathrm{a} \\
(19.1) \\
\end{array}$ & $\begin{array}{r}5.88 \mathrm{e} \\
(-19.9) \\
\end{array}$ & $\begin{array}{c}2.40 \mathrm{~cd} \\
(-24.8)\end{array}$ & $\begin{array}{l}2.24 \text { de } \\
(-29.8)\end{array}$ & $\begin{array}{c}2.83 b \\
(-11.3) \\
\end{array}$ & $\begin{array}{c}0.69 \mathrm{a} \\
(56.8) \\
\end{array}$ & $\begin{array}{l}0.26 \text { ghi } \\
(-40.9)\end{array}$ & $\begin{array}{l}0.48 \mathrm{c} \\
(9.1)\end{array}$ \\
\hline L. aphaca & $\begin{array}{c}11.18 \mathrm{ab} \\
(-7.8)\end{array}$ & $\begin{array}{l}4.40 \mathrm{gh} \\
(-63.7) \\
\end{array}$ & $\begin{array}{l}5.38 \text { ef } \\
(-55.6)\end{array}$ & $\begin{array}{c}4.44 \mathrm{f} \\
(-39.5) \\
\end{array}$ & $\begin{array}{l}6.53 \mathrm{~d} \\
(-11.0) \\
\end{array}$ & $\begin{array}{l}6.66 \mathrm{~cd} \\
(-9.3) \\
\end{array}$ & $\begin{array}{l}2.68 \mathrm{bc} \\
(-16.0)\end{array}$ & $\begin{array}{l}1.60 \mathrm{hi} \\
(-49.8) \\
\end{array}$ & $\begin{array}{c}1.32 \mathrm{ij} \\
(-58.6)\end{array}$ & $\begin{array}{l}0.61 \mathrm{~b} \\
(38.6) \\
\end{array}$ & $\begin{array}{c}0.25 \mathrm{hi} \\
(-48.2) \\
\end{array}$ & $\begin{array}{r}0.20 \mathrm{i} \\
(-54.5) \\
\end{array}$ \\
\hline M. polymorpha & $\begin{array}{l}10.36 \mathrm{~b} \\
(-14.5)\end{array}$ & $\begin{array}{c}7.70 \mathrm{~d} \\
(-36.5)\end{array}$ & $\begin{array}{c}5.33 \text { efg } \\
(-56.0)\end{array}$ & $\begin{array}{c}5.48 \mathrm{e} \\
(-25.3)\end{array}$ & $\begin{array}{l}7.20 \mathrm{bc} \\
(-1.9)\end{array}$ & $\begin{array}{c}3.56 \mathrm{gh} \\
(-51.5)\end{array}$ & $\begin{array}{c}2.11 \mathrm{ef} \\
(-33.9)\end{array}$ & $\begin{array}{c}1.93 \mathrm{fg} \\
(-39.5)\end{array}$ & $\begin{array}{r}1.28 \mathrm{j} \\
(-59.9)\end{array}$ & $\begin{array}{r}0.39 \mathrm{de} \\
(-11.4)\end{array}$ & $\begin{array}{c}0.27 \mathrm{gh} \\
(-38.6)\end{array}$ & $\begin{array}{c}0.36 \mathrm{ef} \\
(-18.2)\end{array}$ \\
\hline LSD at $p \leq 0.05$ & \multicolumn{3}{|c|}{0.965} & \multicolumn{3}{|c|}{0.634} & \multicolumn{3}{|c|}{0.282} & \multicolumn{3}{|c|}{0.057} \\
\hline
\end{tabular}

Means with different letters from each other differ significantly at $\mathrm{p} \leq 0.05$; NS $=$ Non-significant; negative values in parenthesis represent a percent reduction while positive values represent a percent increase over control.

\section{Allelopathic effect of the water extracts of weeds}

Inhibition of germination rate and synchronization, increase in time to germination and inhibition of biomass partitioning in rice were recorded by the allelopathic effect of the water extracts of $T$. polycerata, $V$. sativa, $L$. aphaca and $M$. polymorpha (Tables 1 and 2). The allelopathic inhibition of germination and biomass partitioning in rice might be attributed to the phenolics present in the tissues of these weeds (Zohaib et al., 2014a,b). The causes of inhibition of germination and biomass partitioning in roots and shoots of rice seedlings by the phenolic compounds are relative to the disruption of some vital physiological processes such as respiration, photosynthesis, hormonal activity and cell division and elongation, which hinder the events of germination, plant growth and biomass accumulation partially or fully (Li et al., 2010). 
Many phenolic compounds such as ferulic acid, chlorogenic acid, caffeic acid, vanilic acid, gallic acid, 4-hydroxy-3-methoxybenzoic acid and p-coumaric acid have been reported to cause inhibition of germination and plant growth (Rodzynkiewicz et al., 2006; Muzaffar et al., 2012). Similar results were reported in a bioassay study that revealed the inhibitory allelopathic influence of Sisymbrium irio and Descurainia sophia on germination of wheat at different concentrations of water extracts of shoot, leaf and root. Germination of wheat was inhibited by all the treatments. Most inhibitory effect on germination was caused by the treatment with leaf water extract of both weeds (Edrisi and Farahbakhsh, 2011). Similarly, Sarkar et al. (2012) reported the phytotoxicity of water extracts of leaves, roots and seeds of Cassia tora at different concentrations on the germination of Brassica campestris. Lalmuanpuii and Sahoo (2011) reported a decrease in root and shoot, fresh and dry weights of Zea mays and Oryza sativa in response to plant water extracts of Tectona grandis and Mikania micrantha.

The results of present germination bioassay revealed that water extracts of $V$. sativa and L. aphaca at 5\% concentration caused maximum inhibition of energy of germination; however, the water extracts of the same weeds showed an increase in fresh and dry biomass accumulation in rice roots and shoots (Table 1 and 2). The decreased energy of germination and prolonged time to reach $50 \%$ germination might be attributed to inhibition of respiratory enzymes and enzymes involved in the pentose phosphate pathway in germinating seeds by the phenolics present in water extracts of these weeds (Zohaib et al., 2016). However, increase in biomass accumulation in rice roots and shoots might be due to enhanced photosynthetic activity, increased protein content and improved plant defense system in response to a higher concentration of water soluble phenolics from these weeds over time (Haddadchi and Khorasani, 2006). The results of our study are supported by Otusanya (2008), who reported that leachates of Tithonia diverifolia enhanced photosynthetic pigments (chlorophyll $b$ and total chlorophyll contents) in Lycopersicon esculentum. Similarly, Hegab and Ghareib (2010) reported that the allelochemicals from the C. arvensis methanol extract at various concentrations viz. $75 \mathrm{ppm}, 150 \mathrm{ppm}, 300 \mathrm{ppm}$ and $600 \mathrm{ppm}$ imposed a stimulatory allelopathic effect on the biosynthesis and accumulation of chlorophyll and protein contents, carbohydrates, and phenolic compounds of wheat. Activity of antioxidant enzymes such as peroxidase (POD), catalase (CAT), super oxide dismutase (SOD) and phenol oxidase was increased, while, lipid peroxidation and $\mathrm{H}_{2} \mathrm{O}_{2}$ content in wheat plants was decreased by the effect of allelochemicals. Higher levels of chlorophyll and antioxidants were observed at $300 \mathrm{ppm}$.

In the present study, there was a differential allelopathic influence of the water extracts of the weeds at varying concentrations on rice. A higher concentration of water extracts $(5 \%)$ caused more inhibition of germination and biomass partitioning as compared to a lower concentration $(2.5 \%)$ (Tables 1 and 2). It might be attributed to the higher concentration of allelochemicals present in the water extracts of weeds at the higher extract concentration. Romman et al. (2010) substantiated the leaf water extract of Euphorbia hierosolymitana on wheat at $25 \%, 50 \%, 75 \%$ and $100 \%$ concentrations and reported a substantial decrease in the rate of germination and in fresh and dry weights of roots and shoots of wheat at higher concentrations.

\section{Allelopathic effect of weed residues}

Residues of all leguminous weeds inhibited the synchronization and rate of emergence, delayed the time of emergence, lowered the biomass partitioning and increased the root to shoot ratio of the rice seedlings with different durations of decomposition (Tables 3 and 4). The inhibition of emergence and biomass partitioning might be attributed to the release and accumulation of allelochemicals in the soil from decomposing weed residues (Shaukat et al., 2003). Singh et al. (2005) reported similar results for soil amended with the residues of Parthenium hysterophorus at the rate of $40 \mathrm{~g}$ per $\mathrm{kg}$ soil, which considerably reduced emergence rate, delayed time of emergence and decreased biomass accumulation of B. oleracea, B. rapa and B. campestris. These results were attributed to phenolics released with water from the residues of $P$. hysterophorus in the soil. Similarly, Han et al. (2013) reported that decomposing residues of Allium sativum imposed inhibitory effect on fresh biomass of the root and shoot of Lactuca sativa. Biomass accumulation in roots was higher by the allelopathic influence of weed residues than biomass accumulation in shoots, compared with control, which indicates a higher root/shoot ratio. 
In the present study, there was a differential allelopathic effect by various decomposition durations of different weed residues. The inhibitory effect of residues of $V$. sativa on energy of emergence, root fresh weight and shoot fresh weight was increased with increasing decomposition duration up to 30 days, while inhibition of root dry weight and root/shoot ratio was increased by decomposing residues up to 15 days followed by decrease after residue decomposition for 30 days (Table 3 and 4). The increase in inhibition of emergence and biomass production and partitioning after 30 days might be due to release and accumulation of allelochemicals in the soil with increasing time of residue decomposition, which caused perturbation in water relations and photosynthetic activity of rice plants. By contrast, the decrease in inhibition of root dry weight after 30 days may be attributed to reduced respiration losses in rice roots because of inhibition of respiration by allelochemicals (Narwal et al., 2005). Furthermore, these results indicate that under increased allelopathic activity of allelochemicals, dry matter partitioning to roots may increase in order to enhance root growth and support the plant. However, in the present study, the effect of $T$. polycerata, L. aphaca and $M$. polymorpha on rice emergence was different from that of $V$. sativa in response to duration of residue decomposition (Tables 3 and 4). This might be attributed to differential plant structure and residue decomposition pattern of different plant species (Alam and Shereen, 2002; Bonanomi et al., 2011). Furthermore, the allelopathic effect of $T$. polycerata, L. aphaca and M. polymorpha on rice emergence was decreased after 15 days of decomposition as compared to no decomposition but it increased again after 30 days of decomposition. Nonetheless, there was a similar trend for biomass accumulation and partitioning in rice in response to the allelopathic effect of T. polycerata residues (Tables 3 and 4). In the soil, allelochemicals are decomposed by microbes after they are released from plant body and their activity is reduced with increasing duration but in some cases the decomposition products of allelochemicals become more toxic after some time and hinder the plant growth and development (Albuquerque et al., 2010). In the present study, this might be the reason of decrease in toxicity after residue decomposition for 15 days and increase in toxicity after 30 days. However, in the case of $L$. aphaca and $M$. polymorpha residues, there was a differential response. Inhibition of biomass production and partitioning in rice was increased by increasing decomposition duration of $L$. aphaca and $M$. polymorpha residues from zero to 30 days; that was not the case in rice emergence in response to the allelopathic effect of these weeds, where inhibition was decreased after 15 days of decomposition and increased after 30 days of decomposition (Table 3 and 4). Similarly, Jabeen and Ahmed (2009) found that residues of Asphodelus tenuifolius, Euphorbia hirta and Fumaria indica, mixed thoroughly in the soil had emergence and growth retarding effects on maize seedlings. The allelochemicals released from powder of $A$. tenuifolius and $F$. indica significantly reduced emergence but their effect on plant growth was not consistent with emergence. However, the increase in the inhibitory effect of weed residues on biomass production and accumulation with increasing decomposition duration may be attributed to increased release and accumulation of allelochemicals in the soil over time. An et al. (2001) observed that there was an increase in allelochemical accumulation in the soil by decomposing residues of Vulpia myuros with an increase in decomposition duration.

The results of the present study lead to the conclusion that there was a reduction in germination/emergence rate and synchronization, increase in time of germination/emergence and increase in biomass partitioning to roots as compaered to shoots of rice by the influence of allelochemicals present in the water extracts and residues of leguminous weeds. The inhibitory effect was increased with an increase in extract concentration but there was a differential behavior regarding decomposition duration of residues. Therefore, these weeds must be removed from the field at early growth stages to avoid their damaging effects.

\section{REFERENCES}

Ahmad R., Shaikh A.S. Common weeds of wheat and their control. Pak J Water Res. 2003;7:73-6.

Alam S.M., Shereen A. Effects of plant and weeds residues on the growth of rice. Pak J Bot. 2002;34:139-43.

Albuquerque M.B.D. et al. Allelopathy, an alternative tool to improve cropping systems. A review. Agron Sustain Dev. 2011;31:379-95. 
An M. et al. Phytotoxicity of vulpia residues: IV. Dynamics of allelochemicals during decomposition of vulpia residues and their corresponding phytotoxicity. J Chem Ecol. 2001;27:395-40.

Asaduzzaman M. et al. Allelopathy and allelochemicals in rice weed management. Ban Res Public J. 2010;4:1-14.

Association of Official Seed Analysts - AOSA. Rules for testing seeds. J Seed Technol. 1990;12:1-112.

Bewley J.D., Black M. Seeds: physiology of development and germination. New York: Plenum Press, 1994.

Bonanomi G. et al. Decomposition of Medicago sativa residues affects phytotoxicity, fungal growth and soil-borne pathogen diseases. J Plant Pathol. 2011;93:57-69.

Coolbear P. et al. The effect of low temperature pre sowing treatment under the germination performance and membrane integrity of artificially aged tomato seeds. J Exper Bot. 1984;35:1609-17.

Edrisi S., Farahbakhsh A. Allelopathic effects of Sisymbrium irio L. and Descurainia sophia (L.) Schur on the germination of wheat (Triticum aestivum L.). World Acad Sci Eng Technol. 2011;50:638-40.

Ellis R.A., Roberts E.H. The quantification of ageing and survival in orthodox seeds. Seed Sci Technol. 1981;9:373-409.

Farooq M. et al. Enhancing the performance of direct seeded ûne rice by seed priming. Plant Produc Sci. 2006;9:446-56.

Farooq M. et al. Thermal hardening: a new seed vigor enhancing tool in rice. J Integr Plant Biol. 2005;47:187-93.

Haddadchi G., Khorasani F.M. Allelopathic effects of aqueous extracts of sinapis arvensis on growth and related physiological and biochemical responses of brassica napus. J Sci. 2006;32:23-8.

Hamayun M. et al. Allelopathic effects of Cyperus rotunds and Echinochloa crusgalli on seed germination and plumule and radical growth in maize (Zea mays L.). Pak J Weed Sci Res. 2005;11:81-4.

Han X. et al. Allelopathic effect of decomposed garlic (Allium sativum L.) stalk on lettuce (L. sativa Var. Crispa L.). Pak J Bot. 2013;45:225-33.

Hegab M.M., Ghareib H.R. Methanol extract potential of field bindweed (Convolvulus arvensis L.) for wheat growth enhancement. Inter J Bot. 2010;6:334-42.

Hossain M.K., Alam M.N. Allelopathic effects of Lantana camara leaf extract on germination and growth behavior of some agricultural and forest crops in Bangladesh. Pak J Weed Sci Res. 2010;16:217-26.

Hussain F. et al. Studies on weeds of wheat in Quetta. Pak J Agric Res. 1985;6:1-7.

Jabeen N.; Ahmed, M. Possible allelopathic effects of three different weeds on germination and growth of maize (Zea mays) cultivars. Pak J Bot. 2009;41:1677-83.

Khan R. et al. Bioherbicidal activity of some winter weeds against some crops. Pak J Weed Sci Res. 2012;18:561-9.

Lalmuanpuii R., Sahoo U.K. Allelopathic effects of Tectona grandis L. and Mikania micrantha L. on germination of Zea mays L. and Oryza sativa L. under laboratory condition. Sci Vision, 2011;11:208-13.

Li Z.H. et al. Phenolics and plant allelopathy. Molecules. 2010;15:8933-52.

Lin P.S. et al. Mechanism and active variety of allelochemicals. Acta Bot Sinica. 2004;46:757-66.

Mukhtar I., Bajwa R. Effect of aqueous extracts of some weeds on germination and seedling growth of tomato (Lycopersicon esculentum Mill.). Pak J Phytopathol. 2011;23:138-43.

Muzaffar S. et al. Effect of catechol, gallic acid and pyrogallic acid on the germination, seedling growth and the level of endogenous phenolics in cucumber (Cucumis sativus L.). Inter J Life Sci Biotechnol Pharma Res. 2012;1:50-5.

Narwal S.S. et al. Role of allelopathy in crop production. Herbicides 2005;6:4-23.

Noguchi H.K. Allelochemicals released from rice plants. Jap J Plant Sci. 2008;2:18-25. 
Om H. et al. Allelopathic response of Phalaris minor to crop and weed plants in rice-wheat system. Crop Protect. 2002;21:699-705.

Otusanya O.O. et al. Allelopathoic potential of Tithonia diversifolia (Hemsl) and effect on the germination, growth and cholorophyll accumulation of Capsicum annum L. and Lycopersicon esculentum Mill. Inter J Bot. 2008;4:471-5.

Rice E.L. Allelopathy. 2 ${ }^{\text {nd }}$. ed. London: Academic Press, 1984.

Rodzynkiewicz E.S. et al. The effect of selected phenolic compounds on the initial growth of four weed species. J Plant Diseases Protect. 2006;20:479-86.

Romman S.A. et al. Allelopathic effects of spurge (Euphorbia hierosolymitana) on wheat (Triticum durum). Am Eur J Agric Environ Sci. 2010;7:298-302.

Sarkar E. et al. Allelopathic effect of Cassia tora on seed germination and growth of mustard. Turk J Bot. 2012;36:488-94.

Shaukat S.S. et al. Allelopathic responses of Conyza canadensis (L.) Cronquist: A cosmopolitan weed. Asian J Plant Sci. 2003;2:1034-9.

Singh H.P. et al. Allelopathy in Agroecosystems: An overview. J Crop Prod. 2001;4:1-41.

Singh H.P. et al. Phytotoxic effects of Parthenium hysterophorus residues on three Brassica species. Weed Biol Manage. 2005;5:105-9.

Steel R.G.D. et al. Principles and procedures of statistics: a biometrical approach. $3^{\text {rd }}$ ed. New York: McGraw Hill Book, 1997. p.172-7.

Zohaib A. et al. Influence of water soluble phenolics of Vicia sativa L. on germination and seedling growth of pulse crops. Sci Agric. 2014a;8:148-51.

Zohaib A. et al. Phytotoxic effect of water soluble phenolics from five leguminous weeds on germination and seedling growth of rice. Pak J Weed Sci Res. 2014b;20:417-29.

Zohaib A. et al. Weeds cause losses in field crops through allelopathy. Not Sci Biol. 2016;8:47-56. 